\title{
Fractional Hermite-Hadmard inequalities for convex functions and applications
}

\author{
Muhammad Aslam Noor ${ }^{1}$, Khalida Inayat Noor ${ }^{2}$, Muhammad Uzair Awan ${ }^{3}$ \\ Department of Mathematics,COMSATS Institute of Information Technology, Islamabad, Pakistan \\ E-mail: ${ }^{1}$ noormaslam@gmail.com, ${ }^{2}$ khalidanoor@hotmail.com, ${ }^{3}$ awan.uzair@gmail.com
}

\begin{abstract}
In this paper, we derive a new lemma including third-order derivative of a function via fractional integrals. Using this lemma, we establish some new fractional estimates for Hermite-Hadamard type inequalities for convex functions. Several special cases are also discussed. Some applications to special means of real numbers are also discussed. The ideas and techniques used in this paper may stimulate future investigations regarding Hermite-Hadamard type of inequalities and its application in different areas.
\end{abstract}

2010 Mathematics Subject Classification. 26A33. 26D15, 26A51

Keywords. Convex functions, fractional integrals, Hermite-Hadamard inequality, means.

\section{Introduction}

A convex function $f: I \subseteq \mathbb{R} \rightarrow \mathbb{R}$ with $a<b$ and $a, b \in I$ always obey the following double inequality which is known as Hermite-Hadamard inequality in the literature

$$
f\left(\frac{a+b}{2}\right) \leq \frac{1}{b-a} \int_{a}^{b} f(x) d x \leq \frac{f(a)+f(b)}{2} .
$$

For useful details on Hermite-Hadamard type of integral inequalities, readers are referred to [2]. For some recent investigations on these inequalities, see $[1,3,4,5,7-18]$. In [14] authors proved Hermite-Hadamard type inequalities for fractional integrals. This result inspired many researchers to study and investigate Hermite-Hadmard type of inequalities under the perspective of fractional integrals, for example, see $[8,12,13,16]$.

In this paper, we derive a new result for a three times differentiable function involving fractional integrals. Then, using this result, we obtain several new fractional estimates of Hermite-Hadamard inequalities via convex functions. In last section, we give some applications to special means. We hope that the ideas conveyed in this paper may encourage interested readers to explore new dimensions of research in the field of mathematical inequalities. This is the main motivation of this paper.

\section{Preliminaries}

In this section, we recall some previously known concepts. First of all let set of real numbers be denoted by $\mathbb{R}$. Let $I=[a, b] \subset \mathbb{R}$ be the interval and $I^{\circ}$ be the interior of $I$. We follow these notations throughout the paper unless otherwise specified. 
Definition 2.1. A function $f: I \rightarrow \mathbb{R}$ is said to be classical convex function, if

$$
f((1-t) x+t y) \leq(1-t) f(x)+t f(y), \quad \forall x, y \in I, t \in[0,1] .
$$

Definition $2.2([6])$. Let $f \in L_{1}[a, b]$. Then Riemann-Liouville integrals $J_{a^{+}}^{\alpha} f$ and $J_{b^{-}}^{\alpha} f$ of order $\alpha>0$ with $a \geq 0$ are defined by

$$
J_{a^{+}}^{\alpha} f(x)=\frac{1}{\Gamma(\alpha)} \int_{a}^{x}(x-t)^{\alpha-1} f(t) d t, \quad x>a,
$$

and

$$
J_{b^{-}}^{\alpha} f(x)=\frac{1}{\Gamma(\alpha)} \int_{x}^{b}(t-x)^{\alpha-1} f(t) d t, \quad x<b,
$$

where

$$
\Gamma(\alpha)=\int_{0}^{\infty} e^{-t} x^{\alpha-1} d x
$$

is the Gamma function.

Definition 2.3 ([2]). Recall the following definitions:

1. For arbitrary $a, b \in \mathbb{R} \backslash\{0\}$ and $a \neq b$

$$
L(b, a)=\frac{b-a}{\log b-\log a},
$$

is the logarithmic mean,

2. For arbitrary $a, b \in \mathbb{R}$ and $a \neq b$

$$
A(a, b)=\frac{a+b}{2},
$$

is the arithmetic mean,

3. The extended logarithmic mean $L_{p}$ of two positive numbers $a, b$ is given for $a=b$ by $L_{p}(a, a)=$ $a$ and for $a \neq b$ by

$$
L_{p}(a, b)=\left\{\begin{array}{lr}
{\left[\frac{b^{p+1}-a^{p+1}}{(p+1)(b-a)}\right]^{\frac{1}{p}},} & p \neq-1,0, \\
\frac{b-a}{\log b-\log a}, & p=-1, \\
\frac{1}{e}\left(\frac{b^{b}}{a^{a}}\right)^{\frac{1}{b-a}}, & p=0 .
\end{array}\right.
$$




\section{Main Results}

In this section, we establish our main results. To prove our main results, we need following lemma.

Lemma 3.1. Let $f: I \rightarrow \mathbb{R}$ be three times differentiable function on $I^{\circ}$. If $f^{\prime \prime \prime} \in L[a, b]$, then, following equality for fractional integrals hold:

$$
\begin{aligned}
& \frac{2^{\alpha-1} \Gamma(\alpha+1)}{(b-a)^{\alpha}}\left[J_{\left(\frac{a+b}{2}\right)^{-}}^{\alpha} f(a)+J_{\left(\frac{a+b}{2}\right)^{+}}^{\alpha} f(b)\right]-\frac{(b-a)^{3}}{4(\alpha+1)(\alpha+2)} f^{\prime \prime}\left(\frac{a+b}{2}\right)-f\left(\frac{a+b}{2}\right) \\
& =\frac{(b-a)^{3}}{16(\alpha+1)(\alpha+2)} \int_{0}^{1}(1-t)^{\alpha+2}\left[-f^{\prime \prime \prime}\left(\frac{1+t}{2} a+\frac{1-t}{2} b\right)+f^{\prime \prime \prime}\left(\frac{1-t}{2} a+\frac{1+t}{2} b\right)\right] d t .
\end{aligned}
$$

Proof. Let

$$
\begin{aligned}
I & =\int_{0}^{1}(1-t)^{\alpha+2}\left[-f^{\prime \prime \prime}\left(\frac{1+t}{2} a+\frac{1-t}{2} b\right)+f^{\prime \prime \prime}\left(\frac{1-t}{2} a+\frac{1+t}{2} b\right)\right] d t \\
& =-\int_{0}^{1}(1-t)^{\alpha+2} f^{\prime \prime \prime}\left(\frac{1+t}{2} a+\frac{1-t}{2} b\right) d t+\int_{0}^{1}(1-t)^{\alpha} f^{\prime \prime \prime}\left(\frac{1-t}{2} a+\frac{1+t}{2} b\right) d t \\
& =-I_{1}+I_{2} .
\end{aligned}
$$

Integrating $I_{1}$ on $[0,1]$, we have

$$
\left.\begin{array}{rl}
I_{1}= & \int_{0}^{1}(1-t)^{\alpha+2} f^{\prime \prime \prime}\left(\frac{1+t}{2} a+\frac{1-t}{2} b\right) d t \\
= & \left|-\frac{2(1-t)^{\alpha+2} f^{\prime \prime}\left(\frac{1+t}{2} a+\frac{1-t}{2} b\right)}{b-a}\right|_{0}^{1}-\frac{2(\alpha+2)}{b-a} \int_{0}^{1}(1-t)^{\alpha+1} f^{\prime \prime}\left(\frac{1+t}{2} a+\frac{1-t}{2} b\right) d t \\
= & \frac{2}{b-a} f^{\prime \prime}\left(\frac{a+b}{2}\right)-\frac{2(\alpha+2)}{b-a}\left[\frac{2}{b-a} f^{\prime}\left(\frac{a+b}{2}\right)\right. \\
= & \frac{2}{b-a} f^{\prime \prime}\left(\frac{a+b}{2}\right)-\frac{4(\alpha+2)}{(b-a)^{2}} f^{\prime}\left(\frac{a+b}{2}\right) \\
= & \frac{2}{b-a} f^{\prime \prime}\left(\frac{a+b}{2}\right)-\frac{4(\alpha+2)}{(b-a)^{2}} f^{\prime}\left(\frac{a+b}{2}\right) \\
& +\frac{4(\alpha+1)(\alpha+2)}{(b-a)^{2}}\left[\frac{2}{b-a} f\left(\frac{a+b}{2}\right)-\frac{2^{\alpha+1} \Gamma(\alpha+1)}{(b-a)^{\alpha+1}} J_{\left(\frac{a+b}{2}\right)^{-}}^{\alpha}\left(a+\frac{1-t}{2} b\right) d t\right]
\end{array}\right]
$$




$$
\begin{aligned}
& =\frac{2}{b-a} f^{\prime \prime}\left(\frac{a+b}{2}\right)-\frac{4(\alpha+2)}{(b-a)^{2}} f^{\prime}\left(\frac{a+b}{2}\right) \\
& \quad+\frac{8(\alpha+1)(\alpha+2)}{(b-a)^{3}} f\left(\frac{a+b}{2}\right)-\frac{2^{\alpha+3}((\alpha+1)(\alpha+2)) \Gamma(\alpha+1)}{(b-a)^{\alpha+3}} J_{\left(\frac{a+b}{2}\right)^{-}}^{\alpha} f(a) \\
& =\frac{2}{b-a} f^{\prime \prime}\left(\frac{a+b}{2}\right)-\frac{4(\alpha+2)}{(b-a)^{2}} f^{\prime}\left(\frac{a+b}{2}\right) \\
& \quad+\frac{8(\alpha+1)(\alpha+2)}{(b-a)^{3}} f\left(\frac{a+b}{2}\right)-\frac{2^{\alpha+3} \Gamma(\alpha+3)}{(b-a)^{\alpha+3}} J_{\left(\frac{a+b}{2}\right)^{-}}^{\alpha} f(a) .
\end{aligned}
$$

In a similar way, integrating $I_{2}$ on $[0,1]$, we have

$$
\begin{aligned}
& I_{2}=\int_{0}^{1}(1-t)^{\alpha+2} f^{\prime \prime \prime}\left(\frac{1-t}{2} a+\frac{1+t}{2} b\right) d t \\
& =\left|\frac{2(1-t)^{\alpha+2} f^{\prime \prime}\left(\frac{1-t}{2} a+\frac{1+t}{2} b\right)}{b-a}\right|_{0}^{1}+\frac{2(\alpha+2)}{b-a} \int_{0}^{1}(1-t)^{\alpha+1} f^{\prime \prime}\left(\frac{1-t}{2} a+\frac{1+t}{2} b\right) d t \\
& =-\frac{2}{b-a} f^{\prime \prime}\left(\frac{a+b}{2}\right)+\frac{2(\alpha+2)}{b-a}\left[-\frac{2}{b-a} f^{\prime}\left(\frac{a+b}{2}\right)\right. \\
& \left.+\frac{2(\alpha+1)}{b-a} \int_{0}^{1}(1-t)^{\alpha} f^{\prime}\left(\frac{1-t}{2} a+\frac{1+t}{2} b\right) d t\right] \\
& =-\frac{2}{b-a} f^{\prime \prime}\left(\frac{a+b}{2}\right)-\frac{4(\alpha+2)}{(b-a)^{2}} f^{\prime}\left(\frac{a+b}{2}\right) \\
& +\frac{4(\alpha+1)(\alpha+2)}{(b-a)^{2}} \int_{0}^{1}(1-t)^{\alpha} f^{\prime}\left(\frac{1-t}{2} a+\frac{1+t}{2} b\right) d t \\
& =-\frac{2}{b-a} f^{\prime \prime}\left(\frac{a+b}{2}\right)-\frac{4(\alpha+2)}{(b-a)^{2}} f^{\prime}\left(\frac{a+b}{2}\right) \\
& +\frac{4(\alpha+1)(\alpha+2)}{(b-a)^{2}}\left[-\frac{2}{b-a} f\left(\frac{a+b}{2}\right)+\frac{2^{\alpha+1} \Gamma(\alpha+1)}{(b-a)^{\alpha+1}} J_{\left(\frac{a+b}{2}\right)^{+}}^{\alpha} f(b)\right] \\
& =-\frac{2}{b-a} f^{\prime \prime}\left(\frac{a+b}{2}\right)-\frac{4(\alpha+2)}{(b-a)^{2}} f^{\prime}\left(\frac{a+b}{2}\right) \\
& -\frac{8(\alpha+1)(\alpha+2)}{(b-a)^{3}} f\left(\frac{a+b}{2}\right)+\frac{2^{\alpha+3}((\alpha+1)(\alpha+2)) \Gamma(\alpha+1)}{(b-a)^{\alpha+3}} J_{\left(\frac{a+b}{2}\right)^{+}}^{\alpha} f(b) \\
& =-\frac{2}{b-a} f^{\prime \prime}\left(\frac{a+b}{2}\right)-\frac{4(\alpha+2)}{(b-a)^{2}} f^{\prime}\left(\frac{a+b}{2}\right) \\
& -\frac{8(\alpha+1)(\alpha+2)}{(b-a)^{3}} f\left(\frac{a+b}{2}\right)+\frac{2^{\alpha+3} \Gamma(\alpha+3)}{(b-a)^{\alpha+3}} J_{\left(\frac{a+b}{2}\right)^{+}}^{\alpha} f(b)
\end{aligned}
$$


Summation of (3.2), (3.3) and (3.1) and then multiplying both sides by $\frac{(b-a)^{3}}{16(\alpha+1)(\alpha+2)}$ completes the proof.

Note that for $\alpha=1$ in Lemma 3.1, we have previously known result [18].

Theorem 3.2. Let $f: I \rightarrow \mathbb{R}$ be three times differentiable function on $I^{\circ}$. If $f^{\prime \prime \prime} \in L[a, b]$ and $\left|f^{\prime \prime \prime}\right|$ is convex function, then

$$
\begin{aligned}
& \left|\frac{2^{\alpha-1} \Gamma(\alpha+1)}{(b-a)^{\alpha}}\left[J_{\left(\frac{a+b}{2}\right)^{-}}^{\alpha} f(a)+J_{\left(\frac{a+b}{2}\right)^{+}}^{\alpha} f(b)\right]-\frac{(b-a)^{3}}{4(\alpha+1)(\alpha+2)} f^{\prime \prime}\left(\frac{a+b}{2}\right)-f\left(\frac{a+b}{2}\right)\right| \\
& \leq \frac{(b-a)^{3}}{8(\alpha+1)(\alpha+2)(\alpha+3)}\left[\left|f^{\prime \prime \prime}(a)\right|+\left|f^{\prime \prime \prime}(b)\right|\right] .
\end{aligned}
$$

Proof. Using Lemma 3.1 and the fact that $\left|f^{\prime \prime \prime}\right|$ is convex function, we have

$$
\begin{aligned}
& \left|\frac{2^{\alpha-1} \Gamma(\alpha+1)}{(b-a)^{\alpha}}\left[J_{\left(\frac{a+b}{2}\right)^{-}}^{\alpha} f(a)+J_{\left(\frac{a+b}{2}\right)^{+}}^{\alpha} f(b)\right]-\frac{(b-a)^{3}}{4(\alpha+1)(\alpha+2)} f^{\prime \prime}\left(\frac{a+b}{2}\right)-f\left(\frac{a+b}{2}\right)\right| \\
& =\left|\frac{(b-a)^{3}}{16(\alpha+1)(\alpha+2)} \int_{0}^{1}(1-t)^{\alpha+2}\left[-f^{\prime \prime \prime}\left(\frac{1+t}{2} a+\frac{1-t}{2} b\right)+f^{\prime \prime \prime}\left(\frac{1-t}{2} a+\frac{1+t}{2} b\right)\right] d t\right| \\
& \leq\left|\frac{(b-a)^{3}}{16(\alpha+1)(\alpha+2)} \int_{0}^{1}(1-t)^{\alpha+2} f^{\prime \prime \prime}\left(\frac{1+t}{2} a+\frac{1-t}{2} b\right) d t\right| \\
& +\left|\frac{(b-a)^{3}}{16(\alpha+1)(\alpha+2)} \int_{0}^{1}(1-t)^{\alpha+2} f^{\prime \prime \prime}\left(\frac{1-t}{2} a+\frac{1+t}{2} b\right) d t\right| \\
& \leq \frac{(b-a)^{3}}{16(\alpha+1)(\alpha+2)} \int_{0}^{1}(1-t)^{\alpha+2}\left|f^{\prime \prime \prime}\left(\frac{1+t}{2} a+\frac{1-t}{2} b\right)\right| d t \\
& +\frac{(b-a)^{3}}{16(\alpha+1)(\alpha+2)} \int_{0}^{1}(1-t)^{\alpha+2}\left|f^{\prime \prime \prime}\left(\frac{1-t}{2} a+\frac{1+t}{2} b\right)\right| d t \\
& \leq \frac{(b-a)^{3}}{16(\alpha+1)(\alpha+2)} \int_{0}^{1}(1-t)^{\alpha+2}\left[\left(\frac{1+t}{2}\right)\left|f^{\prime \prime \prime}(a)\right|+\left(\frac{1-t}{2}\right)\left|f^{\prime \prime \prime}(b)\right|\right] d t \\
& +\frac{(b-a)^{3}}{16(\alpha+1)(\alpha+2)} \int_{0}^{1}(1-t)^{\alpha+2}\left[\left(\frac{1-t}{2}\right)\left|f^{\prime \prime \prime}(a)\right|+\left(\frac{1+t}{2}\right)\left|f^{\prime \prime \prime}(b)\right|\right] d t
\end{aligned}
$$




$$
\begin{aligned}
& =\frac{(b-a)^{3}}{16(\alpha+1)(\alpha+2)}\left[\left(\frac{\alpha+5}{(\alpha+3)(\alpha+4)}\right)\left|f^{\prime \prime \prime}(a)\right|+\left(\frac{1}{\alpha+4}\right)\left|f^{\prime \prime \prime}(b)\right|\right] \\
& \quad+\frac{(b-a)^{3}}{16(\alpha+1)(\alpha+2)}\left[\left(\frac{1}{\alpha+4}\right)\left|f^{\prime \prime \prime}(a)\right|+\left(\frac{\alpha+5}{(\alpha+3)(\alpha+4)}\right)\left|f^{\prime \prime \prime}(b)\right|\right] \\
& =\frac{(b-a)^{3}}{8(\alpha+1)(\alpha+2)(\alpha+3)}\left[\left|f^{\prime \prime \prime}(a)\right|+\left|f^{\prime \prime \prime}(b)\right|\right] .
\end{aligned}
$$

This completes the proof.

Q.E.D.

Theorem 3.3. Let $f: I \rightarrow \mathbb{R}$ be three times differentiable function on $I^{\circ}$. If $f^{\prime \prime \prime} \in L[a, b]$ and $\left|f^{\prime \prime \prime}\right|^{q}$ is convex function where $\frac{1}{p}+\frac{1}{q}=1, p, q \geq 1$, then

$$
\begin{aligned}
& \left|\frac{2^{\alpha-1} \Gamma(\alpha+1)}{(b-a)^{\alpha}}\left[J_{\left(\frac{a+b}{2}\right)^{-}}^{\alpha} f(a)+J_{\left(\frac{a+b}{2}\right)^{+}}^{\alpha} f(b)\right]-\frac{(b-a)^{3}}{4(\alpha+1)(\alpha+2)} f^{\prime \prime}\left(\frac{a+b}{2}\right)-f\left(\frac{a+b}{2}\right)\right| \\
& \leq \frac{(b-a)^{3}}{16(\alpha+1)(\alpha+2)}\left(\frac{1}{p(\alpha+2)+1}\right)^{\frac{1}{p}} \\
& \times\left[\left(\frac{3}{4}\left|f^{\prime \prime \prime}(a)\right|^{q}+\frac{1}{2}\left|f^{\prime \prime \prime}(b)\right|^{q}\right)^{\frac{1}{q}}+\left(\frac{1}{2}\left|f^{\prime \prime \prime}(a)\right|^{q}+\frac{3}{4}\left|f^{\prime \prime \prime}(b)\right|^{q}\right)^{\frac{1}{q}}\right] .
\end{aligned}
$$

Proof. Using Lemma 3.1, well known Holder's inequality and the fact that $\left|f^{\prime \prime \prime}\right|^{q}$ is convex function, we have

$$
\begin{aligned}
& \left|\frac{2^{\alpha-1} \Gamma(\alpha+1)}{(b-a)^{\alpha}}\left[J_{\left(\frac{a+b}{2}\right)^{-}}^{\alpha} f(a)+J_{\left(\frac{a+b}{2}\right)^{+}}^{\alpha} f(b)\right]-\frac{(b-a)^{3}}{4(\alpha+1)(\alpha+2)} f^{\prime \prime}\left(\frac{a+b}{2}\right)-f\left(\frac{a+b}{2}\right)\right| \\
& =\left|\frac{(b-a)^{3}}{16(\alpha+1)(\alpha+2)} \int_{0}^{1}(1-t)^{\alpha+2}\left[-f^{\prime \prime \prime}\left(\frac{1+t}{2} a+\frac{1-t}{2} b\right)+f^{\prime \prime \prime}\left(\frac{1-t}{2} a+\frac{1+t}{2} b\right)\right] d t\right| \\
& \leq\left|\frac{(b-a)^{3}}{16(\alpha+1)(\alpha+2)} \int_{0}^{1}(1-t)^{\alpha+2} f^{\prime \prime \prime}\left(\frac{1+t}{2} a+\frac{1-t}{2} b\right) d t\right| \\
& \leq\left|\frac{(b-a)^{3}}{16(\alpha+1)(\alpha+2)} \int_{0}^{1} \frac{(1-t)^{\alpha+2} \mid f^{\prime \prime \prime}}{16(\alpha+1)(\alpha+2)} \int_{0}^{1}(1-t)^{\alpha+2} f^{\prime \prime \prime}\left(\frac{1-t}{2} a+\frac{1+t}{2} b\right) d t\right| \\
& \leq \frac{(b-a)^{3}}{16(\alpha+1)(\alpha+2)} \int_{0}^{1}(1-t)^{\alpha+2}\left|f^{\prime \prime \prime}\left(\frac{1-t}{2} a+\frac{1+t}{2} b\right)\right| d t
\end{aligned}
$$




$$
\begin{aligned}
& \left.\leq \frac{(b-a)^{3}}{16(\alpha+1)(\alpha+2)}\left(\int_{0}^{1}(1-t)^{p(\alpha+2)} d t\right)^{\frac{1}{p}} \int_{0}^{1}\left|f^{\prime \prime \prime}\left(\frac{1+t}{2} a+\frac{1-t}{2} b\right)\right|^{q} d t\right)^{\frac{1}{q}} \\
& +\frac{(b-a)^{3}}{16(\alpha+1)(\alpha+2)}\left(\int_{0}^{1}(1-t)^{p(\alpha+2)} d t\right)^{\frac{1}{p}}\left(\int_{0}^{1}\left|f^{\prime \prime \prime}\left(\frac{1-t}{2} a+\frac{1+t}{2} b\right)\right|^{q} d t\right)^{\frac{1}{q}} \\
& \leq \frac{(b-a)^{3}}{16(\alpha+1)(\alpha+2)}\left(\frac{1}{p(\alpha+2)+1}\right)^{\frac{1}{p}}\left(\frac{3}{4}\left|f^{\prime \prime \prime}(a)\right|^{q}+\frac{1}{2}\left|f^{\prime \prime \prime}(b)\right|^{q}\right)^{\frac{1}{q}} \\
& \quad+\frac{(b-a)^{3}}{16(\alpha+1)(\alpha+2)}\left(\frac{1}{p(\alpha+2)+1}\right)^{\frac{1}{p}}\left(\frac{1}{2}\left|f^{\prime \prime \prime \prime}(a)\right|^{q}+\frac{3}{4}\left|f^{\prime \prime \prime}(b)\right|^{q}\right)^{\frac{1}{q}} \\
& =\frac{(b-a)^{3}}{16(\alpha+1)(\alpha+2)}\left(\frac{1}{p(\alpha+2)+1}\right)^{\frac{1}{p}} \\
& \times\left[\left(\frac{3}{4}\left|f^{\prime \prime \prime}(a)\right|^{q}+\frac{1}{2}\left|f^{\prime \prime \prime}(b)\right|^{q}\right)^{\frac{1}{q}}+\left(\frac{1}{2}\left|f^{\prime \prime \prime}(a)\right|^{q}+\frac{3}{4}\left|f^{\prime \prime \prime}(b)\right|^{q}\right)^{\frac{1}{q}}\right]
\end{aligned}
$$

This completes the proof.

Q.E.D.

Theorem 3.4. Let $f: I \rightarrow \mathbb{R}$ be three times differentiable function on $I^{\circ}$. If $f^{\prime \prime \prime} \in L[a, b]$ and $\left|f^{\prime \prime \prime}\right|^{q}$ is convex function where $q>1$, then

$$
\begin{aligned}
& \left|\frac{2^{\alpha-1} \Gamma(\alpha+1)}{(b-a)^{\alpha}}\left[J_{\left(\frac{a+b}{2}\right)^{\alpha}}^{\alpha} f(a)+J_{\left(\frac{a+b}{2}\right)^{+}}^{\alpha} f(b)\right]-\frac{(b-a)^{3}}{4(\alpha+1)(\alpha+2)} f^{\prime \prime}\left(\frac{a+b}{2}\right)-f\left(\frac{a+b}{2}\right)\right| \\
& \leq \frac{(b-a)^{3}(\alpha+3)}{2^{q+4}(\alpha+1)(\alpha+2)(\alpha+3)(\alpha+4)} \\
& \quad \times\left[\left(\frac{\alpha+5}{\alpha+3}\left|f^{\prime \prime \prime}(a)\right|^{q}+\left|f^{\prime \prime \prime}(b)\right|^{q}\right)^{\frac{1}{q}}+\left(\left|f^{\prime \prime \prime}(a)\right|^{q}+\frac{\alpha+5}{\alpha+3}\left|f^{\prime \prime \prime}(b)\right|^{q}\right)^{\frac{1}{q}}\right] .
\end{aligned}
$$

Proof. Using Lemma 3.1, well known power mean inequality and the fact that $\left|f^{\prime \prime \prime}\right|^{q}$ is convex function, we have

$$
\begin{aligned}
& \left|\frac{2^{\alpha-1} \Gamma(\alpha+1)}{(b-a)^{\alpha}}\left[J_{\left(\frac{a+b}{2}\right)^{-}}^{\alpha} f(a)+J_{\left(\frac{a+b}{2}\right)^{+}}^{\alpha} f(b)\right]-\frac{(b-a)^{3}}{4(\alpha+1)(\alpha+2)} f^{\prime \prime}\left(\frac{a+b}{2}\right)-f\left(\frac{a+b}{2}\right)\right| \\
& =\left|\frac{(b-a)^{3}}{16(\alpha+1)(\alpha+2)} \int_{0}^{1}(1-t)^{\alpha+2}\left[-f^{\prime \prime \prime}\left(\frac{1+t}{2} a+\frac{1-t}{2} b\right)+f^{\prime \prime \prime}\left(\frac{1-t}{2} a+\frac{1+t}{2} b\right)\right] d t\right|
\end{aligned}
$$




$$
\begin{aligned}
\leq & \left|\frac{(b-a)^{3}}{16(\alpha+1)(\alpha+2)} \int_{0}^{1}(1-t)^{\alpha+2} f^{\prime \prime \prime}\left(\frac{1+t}{2} a+\frac{1-t}{2} b\right) d t\right| \\
& +\left|\frac{(b-a)^{3}}{16(\alpha+1)(\alpha+2)} \int_{0}^{1}(1-t)^{\alpha+2} f^{\prime \prime \prime}\left(\frac{1-t}{2} a+\frac{1+t}{2} b\right) d t\right| \\
\leq & \frac{(b-a)^{3}}{16(\alpha+1)(\alpha+2)}\left(\int_{0}^{1}(1-t)^{(\alpha+2)} d t\right)^{1-\frac{1}{q}}\left(\int_{0}^{1}(1-t)^{(\alpha+2)}\left|f^{\prime \prime \prime}\left(\frac{1+t}{2} a+\frac{1-t}{2} b\right)\right|^{q} d t\right)^{\frac{1}{q}} \\
& +\frac{(b-a)^{3}}{16(\alpha+1)(\alpha+2)}\left(\int_{0}^{1}(1-t)^{(\alpha+2)} d t\right)^{1-\frac{1}{q}}\left(\int_{0}^{1}(1-t)^{(\alpha+2)}\left|f^{\prime \prime \prime}\left(\frac{1-t}{2} a+\frac{1+t}{2} b\right)\right|^{q} d t\right)^{\frac{1}{q}} \\
\leq & \frac{(b-a)^{3}}{2^{q+4}(\alpha+1)(\alpha+2)}\left(\frac{1}{\alpha+3}\right)^{1-\frac{1}{q}}\left(\frac{\alpha+5}{(\alpha+3)(\alpha+4)}\left|f^{\prime \prime \prime}(a)\right|^{q}+\frac{1}{\alpha+4}\left|f^{\prime \prime \prime}(b)\right|^{q}\right)^{\frac{1}{q}} \\
& +\frac{(b-a)^{3}}{2^{q+4}(\alpha+1)(\alpha+2)}\left(\frac{1}{\alpha+3}\right)^{1-\frac{1}{q}}\left(\frac{1}{\alpha+4}\left|f^{\prime \prime \prime}(a)\right|^{q}+\frac{\alpha+5}{(\alpha+3)(\alpha+4)}\left|f^{\prime \prime \prime}(b)\right|^{q}\right)^{\frac{1}{q}} \\
= & \frac{(b-a)^{3}(\alpha+3)}{2^{q+4}(\alpha+1)(\alpha+2)(\alpha+3)(\alpha+4)} \\
& \times\left[\left(\frac{\alpha+5}{\alpha+3}\left|f^{\prime \prime \prime}(a)\right|^{q}+\left|f^{\prime \prime \prime}(b)\right|^{q}\right)^{\frac{1}{q}}+\left(\left|f^{\prime \prime \prime}(a)\right|^{q}+\frac{\alpha+5}{\alpha+3}\left|f^{\prime \prime \prime}(b)\right|^{q}\right)\right. \\
& {\left[\frac{1}{q}\right] }
\end{aligned}
$$

This completes the proof.

Q.E.D.

\section{Some Applications to special means}

In this section, we present some applications to means of real numbers.

Proposition 4.1. For some $n \in \mathbb{Z}\{-1,0\}, 0 \leq a<b$, then

$$
\begin{aligned}
& \left|L(a, b)-\frac{n(n-1)(b-a)^{3}}{24} A^{n-2}(a, b)-A^{n}(a, b)\right| \\
& \leq \frac{n(n-1)(n-2)(b-a)^{3}}{192}\left[|a|^{n-3}+|b|^{n-3}\right] .
\end{aligned}
$$

Proof. The assertion directly follows from Theorem 3.2 applying for $f(x)=x^{n}$ and $\alpha=1$. $\quad$ Q.E.D. 
Proposition 4.2. For some $n \in \mathbb{Z}\{-1,0\}, 0 \leq a<b$ and $\frac{1}{p}+\frac{1}{q}=1,1<q<\infty$, then

$$
\begin{aligned}
& \left|L(a, b)-\frac{n(n-1)(b-a)^{3}}{24} A^{n-2}(a, b)-A^{n}(a, b)\right| \\
& \leq \frac{n(n-1)(n-2)(b-a)^{3}}{96}\left(\frac{1}{3 p+1}\right)^{\frac{1}{p}} \\
& \quad \times\left[\left(\frac{3}{4}|a|^{q(n-3)}+\frac{1}{2}|b|^{q(n-3)}\right)^{\frac{1}{q}}+\left(\frac{1}{2}|a|^{q(n-3)}+\frac{3}{4}|b|^{q(n-3)}\right)^{\frac{1}{q}}\right] .
\end{aligned}
$$

Proof. The assertion directly follows from Theorem 3.3 applying for $f(x)=x^{n}$ and $\alpha=1$. Q.E.D.

Proposition 4.3. For some $n \in \mathbb{Z}\{-1,0\}, 0 \leq a<b$ and $q>1$, then

$$
\begin{aligned}
& \left|L(a, b)-\frac{n(n-1)(b-a)^{3}}{24} A^{n-2}(a, b)-A^{n}(a, b)\right| \\
& \leq \frac{n(n-1)(n-2)(b-a)^{3}}{384}\left(\frac{4}{5}\right)^{\frac{1}{q}} \\
& \quad \times\left[\left(\frac{3}{2}|a|^{q(n-3)}+|b|^{q(n-3)}\right)^{\frac{1}{q}}+\left(|a|^{q(n-3)}+\frac{3}{2}|b|^{q(n-3)}\right)^{\frac{1}{q}}\right] .
\end{aligned}
$$

Proof. The assertion directly follows from Theorem 3.4 applying for $f(x)=x^{n}$ and $\alpha=1$. Q.E.D.

Proposition 4.4. For some $0 \leq a<b$, then

$$
\left|L^{-1}(a, b)-\frac{(b-a)^{3}}{12} A^{-3}(a, b)-A^{-1}(a, b)\right| \leq \frac{(b-a)^{3}}{32}\left[|a|^{-4}+|b|^{-4}\right] .
$$

Proof. The assertion directly follows from Theorem 3.2 applying for $f(x)=x^{-1}$ and $\alpha=1$. Q.E.D.

Proposition 4.5. For some $0 \leq a<b$ and $\frac{1}{p}+\frac{1}{q}=1,1<q<\infty$, then

$$
\begin{aligned}
& \left|L^{-1}(a, b)-\frac{(b-a)^{3}}{12} A^{-3}(a, b)-A^{-1}(a, b)\right| \\
& \leq \frac{(b-a)^{3}}{16}\left(\frac{1}{3 p+1}\right)^{\frac{1}{p}}\left[\left(\frac{3}{4}|a|^{-4 q}+\frac{1}{2}|b|^{-4 q}\right)^{\frac{1}{q}}+\left(\frac{1}{2}|a|^{-4 q}+\frac{3}{4}|b|^{-4 q}\right)^{\frac{1}{q}}\right] .
\end{aligned}
$$

Proof. The assertion directly follows from Theorem 3.3 applying for $f(x)=x^{-1}$ and $\alpha=1$. Q.E.D.

Proposition 4.6. For some $0 \leq a<b$ and $q>1$, then

$$
\begin{aligned}
& \left|L^{-1}(a, b)-\frac{(b-a)^{3}}{12} A^{-3}(a, b)-A^{-1}(a, b)\right| \\
& \leq \frac{(b-a)^{3}}{64}\left(\frac{4}{5}\right)^{\frac{1}{q}}\left[\left(\frac{3}{2}|a|^{-4 q}+|b|^{-4 q}\right)^{\frac{1}{q}}+\left(|a|^{-4 q}+\frac{3}{2}|b|^{-4 q}\right)^{\frac{1}{q}}\right] .
\end{aligned}
$$


Proof. The assertion directly follows from Theorem 3.4 applying for $f(x)=x^{-1}$ and $\alpha=1$. Q.E.D.

\section{Acknowledgements}

The authors would like to thank Dr. S. M. Junaid Zaidi, Rector, COMSATS Institute of Information Technology, Pakistan, for providing excellent research and academic environment.

\section{References}

[1] G. Cristescu, Improved integral inequalities for products of convex functions, J. Ineq. Pure Appl. Math. 6(2), (2005).

[2] S. S. Dragomir, C. E. M. Pearce, Selected topics on Hermite-Hadamard inequalities and applications, Victoria University, Australia (2000).

[3] S. S. Dragomir, J. Pečarić, L. E. Persson, Some inequalities of Hadamard type, Soochow J. Math, 21, 335-341, (1995).

[4] S.-H. Wang, B.-Y. Xi, F. Qi, Some new inequalities of Hermite-Hadamard type for $n$-time differentiable functions which are m-convex, Analysis 32, 247-262 (2012)/DOI 10.1524/anly.2012.1167.

[5] W.-D. Jiang, D.-W. Niu, Y. Hua, F. Qi, Generalizations of Hermite-Hadamard inequality to $n$-time differentiable functions which are s-convex in the second sense, Analysis 32, 209-220 (2012)/DOI 10.1524/anly.2012.1161.

[6] A. Kilbas, H. M. Srivastava, J. J. Trujillo, Theory and applications of fractional differential equations, Elsevier B.V., Amsterdam, Netherlands, (2006).

[7] S. K. Khattri, Three proofs of the inequality $e<\left(1+\frac{1}{n}\right)^{n+0.5}$, Amer. Math. Monthly, 117(3) (2010), 273-277.

[8] M. A. Noor, M. U. Awan, Some integral inequalities for two kinds of convexities via fractional integrals, Trans. J. Math. Mech. 5(2), (2013), 129-136.

[9] M. A. Noor, M. U. Awan, K. I. Noor, On some inequalities for relative semi-convex functions, J. Ineq. Appl. 2013, 2013:332.

[10] M. A. Noor, K. I. Noor, M. U. Awan, Geometrically relative convex functions, Appl. Math. Inform. Sci, 8(2), (2014), 607-616.

[11] M. A. Noor, K. I. Noor, M. U. Awan, Hermite-Hadamard inequalities for relative semi-convex functions and applications, Filomat, 28(2)(2014), 221-230.

[12] M. A. Noor, G. Cristescu, M. U. Awan, Generalized fractional Hermite-Hadamard inequalities for twice differentiable s-convex functions, Filomat, 29(4), (2015), 807-815. 
[13] M. E. Ozdemir, H. Kavurmaci, C Yildiz, Fractional integral inequalities via s-convex functions, available online at: arXiv:1201.4915v1, (2012).

[14] M. Z. Sarikaya, E. Set, H. Yaldiz, N. Basak, HermiteHadamards inequalities for fractional integrals and related fractional inequalities, Math. Comp. Modelling 57, 2403-2407, 2013.

[15] Y. Shuang, H.-P. Yin, F. Qi, HermiteHadamard type integral inequalities for geometricarithmetically s-convex functions, Analysis 33, 197208 (2013)/DOI 10.1524/anly.2013.1192.

[16] J. Wang, X. Li, M. Feckan, Y. Zhou, Hermite-Hadamard-type inequalities for Riemann-Liouville fractional integrals via two kinds of convexity, Appl. Anal. 2012, http://dxdoi.org/10.1080/00036811.2012.727986.

[17] B.-Y. Xi, F. Qi, Some integral inequalities of Hermite-Hadamard type for convex functions with applications to means, J. Func. Spaces Appl., 2012(2012), Article ID 980438.

[18] B.-Y Xi, S.-H Wang, F. Qi, Some inequalities of Hermite-Hadamard type for functions whose 3rd derivatives are P-convex, Appl. Math., 3, 1898-1902, (2012). 